\title{
Proposta de um método para escolha de software de automação de bibliotecas ${ }^{1}$
}

\author{
Lígia Café \\ Doutora em Lingüística. Instituto Brasileiro de Informação em Ciên- \\ cia e Tecnologia (IBICT).
}

\author{
Christophe Dos Santos \\ Bacharel em documentação e informação científica e técnica (DIST). \\ Université Claude-Bernard (Lyon, France).
}

\section{Flávia Macedo}

Bibliotecária do Conselho da Justiça Federal. Conselho da Justiça Federal.

\section{Resumo}

Apresenta um método para avaliação e seleção de softwares de automação de bibliotecas. Consiste na atribuição de critérios e cálculos estatísticos em uma lista elaborada para a seleção e avaliação deste tipo de software. Este método pretender servir como instrumento de apoio à tomada de decisão no processo de escolha do software mais adequado às necessidades de cada instituição. Este trabalho foi motivado por uma demanda do Instituto Brasileiro de Informação em Ciência e Tecnologia (IBICT) para automatizar a sua biblioteca.

\section{Palavras-chave}

Automação de bibliotecas; Software para automação de bibliotecas; Método estatístico; Avaliação de software.

\section{Proposed method for selecting library automation software}

\begin{abstract}
This paper presents a method for evaluation and selection of library automation software. The method intends to apply specific statistical criteria and calculus to a checklist constructed for the selection and evaluation of automationrelated software. It aims to serve as an instrument of support to the decision-making process of choosing a software to better serve the needs of each library. This paper was motivated by a demand of the Brazilian Institute of Information in Science and Technology (IBICT) to automate its library.
\end{abstract}

\section{Keywords}

Library automation; Library automation software; Statistical method; Software evaluation.

\section{INTRODUÇÃO}

A chegada da informática provocou profundas mudanças no conceito de organização e funcionamento das bibliotecas. $O$ sucesso da informatização não foi fruto apenas de uma transposição de esquemas físicos. Ele foi o resultado de uma reflexão sobre as velhas estruturas com vistas a formar uma nova organização. Desta forma, os antigos esquemas de tratamento do livro (ou de outros suportes) foram revistos com o objetivo de conceber um processamento integrado e eficiente.

Nos países desenvolvidos, as bibliotecas se automatizaram durante os anos 80 e começo dos anos 90. No início deste período, foram as grandes bibliotecas que tomaram a frente o processo de automação de seus acervos. Era a época em que os sistemas eram construídos com o objetivo de resolver problemas específicos de uma biblioteca em particular. Posteriormente, surgiram softwares prontos para serem utilizados. Não era mais a biblioteca que ditava suas necessidades, mas as empresas detentoras do produto que ofereciam sistemas nos quais vinham embutidas soluções para a automação da biblioteca como um todo. Se, por um lado, estes pacotes tinham de sofrer adaptações que nem sempre eram possíveis de serem concretizadas, por outro, esta nova era permitiu que bibliotecas de menor porte pudessem adquirir pacotes mais acessíveis financeiramente, iniciando assim a sua automação.

Na literatura estrangeira, o tema automação de bibliotecas atingiu seu ápice no final dos anos 80. Em uma consulta ao índice do Annual Review of Information Science and Technology (Arist), verificou-se que uma das últimas revisões publicadas sobre o assunto data de 1987 (Shaw \& Culkin, 1987). Neste número, Shaw \& Culkin apresentavam o novo panorama que se delineava na época e discutiam as transformações dos sistemas de automação de bibliotecas e os serviços de informação baseados em computador. Estes autores ressaltavam o foco no usuário final e a criação de redes que facilitavam o acesso à informação, tornando a biblioteca local o principal

\footnotetext{
${ }^{1}$ Gostaríamos de agradecer a Elza Maria Ferraz Barbosa e toda a equipe que participou do projeto. Em especial, a Regina Márcia de Castro Silva e Maria Estefânia Ponte Pinheiro, que muito nos incentivaram a publicar este estudo.
} 
provedor de informação. Era a época em que se discutia o contraponto entre os conceitos de automação de biblioteca e automação da informação. No primeiro, o processo de automação era visto como o de tornar a biblioteca um ambiente onde fosse possível identificar a disponibilidade de qualquer documento no acervo por meio de uma pergunta ao sistema e gerar relatórios e estatísticas relativas ao uso da coleção. Isso sem perder de vista a noção de que a automação das diversas atividades da biblioteca deveria estar integrada em um ambiente de comunicação. No segundo conceito, a automação estava voltada para a concepção de sistemas de busca de informação (search systems). Para muitos, estes dois conceitos eram tratados como estágios da automação de bibliotecas.

Atualmente, no Brasil, as bibliotecas começam a se automatizar e ao mesmo tempo a rever sua organização para se adaptarem melhor às novas tecnologias colocadas à disposição deste mercado. A escolha do melhor software de automação nem sempre é feita de maneira sistemática. Conscientes da complexidade desta nova realidade que se delineia no contexto brasileiro, propomos um método que auxilie a escolha de um software mais adequado às necessidades da biblioteca que procura se automatizar.

\section{MOTIVAÇÃO}

Este trabalho foi motivado por uma demanda do Instituto Brasileiro de Informação em Ciência e Tecnologia (Ibict) para automatizar a sua biblioteca. A automação envolve uma série de tomadas de decisões anteriores à escolha do software. É preciso tomar cuidado com decisões baseadas em ideologias, modismos e expectativas pessoais ou ainda em argumentos feitos de acordo com situações específicas, sem observar a biblioteca como um todo. Para tanto, devem ser privilegiadas as análises sistemáticas de fatos reais baseadas em métodos comparativos.

A equipe ${ }^{2}$ destinada a conduzir o projeto de automação da biblioteca iniciou o trabalho levantando na literatura diversos artigos que traziam listas de critérios de avaliação de softwares de automação de bibliotecas. Estes critérios se apresentavam em igual ordem de relevância, sem indicação de juízo de valor em relação à realidade da biblioteca. A falta de atribuição de níveis de importância para cada critério impedia seleção precisa daqueles itens que seriam mais exigidos no momento de avaliar um software. Além disso, muitos autores apresentavam vantagens e desvantagens de alguns softwares sem especificar as condições em que estes sistemas foram aplicados.

$\mathrm{Na}$ medida em que cada biblioteca tem suas próprias necessidades em relação ao tipo de automação que deseja implementar, a simples consulta a listas de critérios pode não ser suficiente. Tendo em vista esta problemática, a equipe decidiu reunir os critérios levantados na literatura e elaborar um método estatístico de avaliação baseado em pesos e notas para cada critério. $\mathrm{O}$ modelo proposto traz como inovação a possibilidade de ser adaptado à realidade de diversos tipos de bibliotecas.

\section{METODOLOGIA}

A metodologia para avaliação e seleção de software para automação de bibliotecas seguiu os seguintes passos:

\section{1) Revisão da literatura sobre automação de bibliotecas}

Nesta etapa foram consultados vários documentos que relatavam diversas experiências de escolha do software adequado para automatizar bibliotecas. Desta literatura, foram retirados os critérios de avaliação considerados mais relevantes. $\mathrm{O}$ estudo baseou-se principalmente na lista apresentada no documento Avaliação de softwares para bibliotecas (Côrte \& Almeida, 2000), ao qual foram acrescentados outros critérios julgados pertinentes. Esta primeira lista foi então discutida pelo grupo e modificada à medida que novas sugestões foram sendo apresentadas. No final desta etapa, foi definida uma lista definitiva que continha 181 critérios (ver anexo). Cabe ressaltar que estes critérios, apesar de terem sido elaborados para resolver a automação de uma biblioteca específica, foram selecionados com a preocupação de apontar aspectos gerais e assim atender a um espectro maior de avaliações desta natureza. Desta forma, qualquer biblioteca poderá utilizar esta lista como ponto de partida, fazendo suas adaptações caso julgue necessário.

O quadro 1, a seguir, apresenta as dez grandes categorias de avaliação e o número de critérios contidos em cada uma delas ${ }^{3}$.

\footnotetext{
${ }^{2}$ Esta equipe era formada pelos três autores deste artigo e mais três pessoas da área da informática e cinco pessoas da biblioteca.
}

${ }^{3}$ A lista na sua íntegra encontra-se em anexo. 
Um dos objetivos que norteou todo o processo de elaboração desta lista foi o de construir um instrumento simples, de fácil utilização e ao mesmo tempo o mais completo possível.

2) Elaboração de um sistema de notação com atribuição de pesos e notas aos critérios

Uma vez que a lista foi validada por toda a equipe, foi elaborado um sistema de notação. Por meio da atribuição de pesos e notas, o sistema nos permite fazer uma distinção precisa entre critérios de relevância variável.

Descrevemos a seguir o método utilizado.

\subsection{Os pesos}

Foram estabelecidos três pesos para cada critério:

- Indispensável - coeficiente (3). Este peso é atribuído a um critério imprescindível. Exemplo: a integração de todas as funções de uma biblioteca é indispensável. Sem ela, a automação da biblioteca não pode ser completada.

- Importante - coeficiente (2). Este peso é atribuído a um critério que pode ser interessante para a biblioteca. Exemplo: o controle do orçamento da aquisição é interessante, mas não é fundamental para a realização das tarefas de seleção e aquisição.

- Dispensável - coeficiente (1). Este peso é atribuído a um critério que é considerado desnecessário para a biblioteca. Exemplo: a possibilidade de imprimir etiquetas para bolso de livro é desnecessária uma vez que o empréstimo utilizará código de barras.

Uma vez determinada esta notação, a lista de critérios foi distribuída com as instruções para atribuição do peso considerado adequado ao tipo de critério analisado. Vale ressaltar que as pessoas que preencheram a planilha se detiveram apenas a análise do conjunto de critérios de sua competência. Assim, o pessoal da área de informática, por exemplo, analisou somente o conjunto de critérios relativos às características gerais do software, ergonomia e tecnologia.

Quando todos os formulários foram devolvidos devidamente preenchidos, foi calculado para cada critério um peso médio P. Foi determinado também o desvio padrão (ss), o que confirmou a validade de 176 pesos atribuídos aos 181 critérios. Na verdade, sobre 181 pesos médios, cinco possuíam um desvio padrão acima de 0.8. Este valor indica significativa divergência de opinião na atribuição de pesos (ou seja, para um mesmo peso uma pessoa atribuiu um peso máximo e outra um peso mínimo). No entanto,
QUADRO 1

Número de critérios por categoria de avaliação

\begin{tabular}{lc}
\hline \multicolumn{1}{c}{ Categoria de avaliação } & $\begin{array}{c}\text { Número de } \\
\text { critérios } \\
\text { inseridos }\end{array}$ \\
Características gerais do software & 7 \\
Ergonomia & 3 \\
Tecnologia (hardware, compatibilidade e rede) & 25 \\
Seleção e aquisição & 22 \\
Processamento técnico & 20 \\
Circulação & 10 \\
Recuperação da informação & 57 \\
Disseminação da informação & 2 \\
Processo gerencial & 19 \\
Características da empresa fornecedora & 16 \\
\hline
\end{tabular}

esta divergência foi verificada em apenas $2.8 \%$ das respostas. Além disso, foi possível constatar que três desses casos se encontravam no conjunto de critérios sobre tecnologia, o que se explica em grande parte por uma má interpretação ou explicação incorreta dos critérios. Por exemplo, o critério < acesso ilimitado de usuários > poderia ter sido, por um lado, negligenciado se o respondente considerasse que é a capacidade da rede que determina em grande parte este aspecto. Por outro lado, este critério poderia ter sido julgado indispensável se fosse considerada a capacidade do software para gerenciar todas estas sessões.

Observando os 171 critérios cujo desvio padrão foi menor que 0.8 , foi possível concluir que houve grande homogeneidade no total de atribuições de pesos. Isto é, $7.2 \%$ das respostas apresentaram pouca divergência de opinião. Este resultado poderia ser justificado pelo fato de que as pessoas que responderam o formulário tinham conhecimento das necessidades da biblioteca e domínio sobre o tema automação.

\subsection{As notas}

Após finalizada a etapa de atribuição de pesos, foi proposta uma escala de notas. Estas notas seriam atribuídas no momento da avaliação de cada software. Elas obedecem a uma escala de valor de 0 a 5 , sendo assim distribuídas :

- Inexistente (0) - o critério não existe. Por exemplo: se o software não oferece a possibilidade de fazer uma reserva em linha, este critério receberá a nota (0).

- Muito ruim (1) - o critério possui uma série de problemas, como, por exemplo, apresentar uma interface de difícil utilização. Este critério receberá nota (1). 
- Ruim (2) - o critério possui ainda pequenos problemas. Este critério receberá nota (2).

- Regular (3) - o critério funciona. Este critério receberá nota (3).

- Bom (4) - o critério funciona perfeitamente e oferece algumas facilidades de utilização. Este critério receberá nota (4).

- Excelente (5) - o critério funciona perfeitamente e oferece opções suplementares interessantes. Este critério receberá nota $(5)$.

Definimos propositadamente uma escala par de seis notas, pois consideramos que a inexistência de uma nota central (como seria o caso de uma escala ímpar) força uma escolha mais consciente. Com uma escala ímpar do tipo $1-2-3$ $-4-5$, é maior a tendência a atribuir a nota central (3) quando o respondente não compreende bem o critério, o que poderia falsear os resultados aproximando-os artificialmente da média.

\section{3) Cálculo da nota final}

Com o objetivo de consolidar as notas e pesos atribuídos, utilizamos a seguinte fórmula para calcular a nota final para cada critério indicado:

$$
\mathrm{F}=\mathrm{N} \times \mathrm{P}
$$

onde,

$\mathrm{F}=$ nota final do critério avaliado

$\mathrm{N}=$ média da nota atribuída a um critério

$\mathrm{P}=$ média do peso atribuído a um critério

Esta nota terá um valor entre 0 e 15 para cada critério. A soma de todas as notas finais $(\mathrm{F})$ representará a avaliação final do software pela equipe.

\section{CONCLUSÃO}

A decisão por um software que automatize de forma eficiente uma biblioteca não é uma tarefa fácil. A variedade de opções existentes aliada às peculiaridades de cada biblioteca dificulta ainda mais a escolha.

Devido a essa complexidade, a estratégia de avaliação e seleção do novo sistema requer a elaboração de um projeto específico e detalhado. Um projeto de automação de bibliotecas deve contemplar as seguintes etapas, sugeridas pela literatura: 1) definição dos objetivos da automação; 2) diagnóstico da biblioteca, que inclui a identificação das diretrizes organizacionais, o levantamento de dados bibliográficos e de infra-estrutura, entre outros, a média de crescimento do acervo, os produtos e serviços oferecidos etc.; 3) elaboração de fluxogramas das rotinas para análise dos procedimentos atuais; 4) identificação das necessidades da biblioteca a partir das informações coletadas; 5) levantamento de informações sobre os softwares disponíveis e agendamento das demonstrações com as empresas; 6) análise das demonstrações e instalação das versões de teste dos produtos selecionados para avaliação.

Sugerimos neste artigo um método de avaliação baseado em critérios e cálculos estatísticos, que procura auxiliar a tomada de decisão quanto à melhor escolha a ser feita. $\mathrm{Na}$ aplicação do método, o diagnóstico deve ser a base para a elaboração de critérios de avaliação e para a atribuição de pesos, pois é ele que retrata as reais necessidades da instituição.

Mesmo que seja pouco provável que se encontre um sistema perfeito, a tendência verificada nos países desenvolvidos é a compra de sistemas comerciais, sendo raras as instituições que adquirem softwares elaborados sob encomenda para a biblioteca. Na verdade, o sistema que realmente responderá às necessidades de automação deverá ser alcançado por meio da customatização, critério fundamental na escolha de um software. Além disso, de acordo com Rowley (1990), os sistemas comerciais podem se aperfeiçoar com a experiência adquirida a partir das demandas específicas de cada biblioteca. A troca de informações com instituições que testaram ou adquiriram o produto vem enriquecer ainda mais as possibilidades de se obter um sistema satisfatório.

Rowley (1990) afirma ainda que é importante verificar a estabilidade do fornecedor no mercado, para evitar futuros problemas que levem à substituição do sistema antes do tempo. Millard (1999) salienta que, sendo a compra de um novo sistema um grande investimento, o software adquirido deve satisfazer às necessidades da instituição no período de pelo menos cinco a oito anos, com as devidas atualizações.

Segundo Buckland (1992), as bibliotecas podem ser classificadas em três tipos, considerando sua evolução tecnológica : a) bibliotecas tradicionais, cujo acervo é composto essencialmente de documentos em suporte de papel e outros (como os microfilmes); b) bibliotecas automatizadas, cujo processamento técnico é feito pelo 
computador, mas o suporte dos documentos permanece em papel; c) bibliotecas eletrônicas ou digitais, cujo tratamento do material é feito pelo computador e o acervo se encontra em suporte eletrônico. Seguindo esta classificação, pode-se dizer que atualmente no Brasil existe uma realidade híbrida, em que a maioria das bibliotecas é do tipo tradicional ou se encontra em fase de automação. No entanto, já é possível verificar iniciativas no campo das bibliotecas eletrônicas ou digitais.

Como afirma Buckland (1992), o surgimento das bibliotecas eletrônicas ou digitais, onde os documentos são armazenados e disseminados em meio eletrônico, está provocando o redimensionamento das formas de prestação de serviços de informação. Segundo este autor, essa nova fase traz consigo uma mudança conceitual. Enquanto a automação das tarefas de processamento interno da biblioteca facilita o trabalho dos bibliotecários, a biblioteca eletrônica parece mais revolucionária do ponto de vista do usuário, uma vez que documentos eletrônicos podem ser utilizados de qualquer lugar e a qualquer hora.

O foco no usuário foi um processo evolutivo e, como lembra Heseltine (1995), começou com a disponibilização dos catálogos públicos de acesso em linha (OPAC's) e com a utilização de bases de dados em cd-rom para pesquisa. A partir daí, a interface dos sistemas passou a ter um papel extremamente significativo.

Apesar da transição tardia, as bibliotecas brasileiras que começam a se automatizar têm a possibilidade de ultrapassar algumas etapas de evolução tecnológica, podendo contar com tecnologias muito mais avançadas do que aquelas disponíveis há duas décadas. Por este motivo, todas as tendências do mercado atual devem ser levadas em consideração para a escolha do software de automação. Devemos estar informados e preparados para receber as novas tecnologias e fugir da obsolescência.

Estar preparado para o futuro significa, por exemplo, dar a devida importância aos padrões adotados pelas grandes redes de bibliotecas no mundo. Os padrões facilitam a automação e aumentam o custo-benefício dos processos automatizados. De acordo com Buckland (1992), dois padrões são de grande importância para a automação de bibliotecas. $\mathrm{O}$ primeiro deles é o formato MARC, que torna os dados bibliográficos legíveis por computador, possibilitando seu compartilhamento por meio de diferentes sistemas (Furrie, 2000). O segundo padrão é o protocolo de pesquisa e recuperação de informações Z39.50, que permite, por exemplo, acessar simultaneamente os catálogos da biblioteca local e das maiores bibliotecas do mundo a partir da mesma interface.
Na nova geração de sistemas, os padrões são essenciais para garantir maior eficiência e conectividade. $\mathrm{O}$ uso do formato MARC, por exemplo, garante que as bibliotecas substituam seu sistema de automação por outro com a segurança de que os dados armazenados pelo antigo sistema não sejam perdidos na conversão retrospectiva. O protocolo Z39.50, por sua vez, passou a ser compatível com o formato MARC devido à expansão de seu uso em bibliotecas. Isso significa que os resultados de uma pesquisa em diversas bibliotecas podem ser retornados em MARC e podem ser utilizados para catalogação cooperativa (Miller, 1999). Alguns produtos disponíveis no mercado nacional já oferecem opções de software compatíveis com o formato MARC e com o protocolo Z39.50.

Os documentos eletrônicos também exigem padrões de formato, de catalogação, de transferência. A biblioteca eletrônica irá envolver softwares e hardwares heterogêneos, o que requer grande ênfase em compatibilidade, comunicação e interoperabilidade por meio de protocolos padronizados. Em suma, os padrões deverão ser adotados tanto em nível nacional como internacional.

Finalmente, cabe ressaltar que todo este elenco de preocupações deve ser levado em consideração sem se perder de vista que a escolha do software de automação deve basear-se essencialmente no propósito central das bibliotecas: a prestação de serviços de acesso à informação. Este propósito envolve o armazenamento, a identificação, a localização e a disponibilização de um documento da maneira mais eficiente possível. 


\section{REFERÊNCIAS BIBLIOGRÁFICAS}

BUCKLAND, Michael. Redesigning library services: a manifesto. Chicago: American Library Association, 1992. Disponível em: <http:// sunsite.berkeley.edu/Literature/Library/Redesigning/html.html $>$. Acesso em: 25 ago. 2000.

CÔRTE, A. R. E; ALMEIDA, I. M. de. (Coord.). Avaliação de softwares para bibliotecas. São Paulo: Polis, 2000. 108 p.

CÔRTE, A. R. E et al. Automação de bibliotecas e centros de documentação: o processo de avaliação e seleção de softwares. Ciência da Informação, Brasília, v. 28, n. 3, p. 241-256, set./dez. 1999.

FURRIE, Betty. Understanding MARC bibliographic: machine-readable cataloging. 5.ed. Washington, DC: Library of Congress, 2000. Disponível em: <http://lcweb.loc.gov/marc/umb/>. Acesso em: 25 ago. 2000.

HESELTINE, Richard. Follett WP new directions in the library automation industry: prospects for higher education. [S. 1. : s. n.,1995]. Disponível em: <http://www.niss.ac.uk/education/hefc/follett/wp/18.html>. Acesso em: 26 ago. 2000.

LAM, K.T. Planning for library automation. [S. 1. : s. n., 2000]. Disponível em: <http://ihome.ust.hk/ lblkt/libauto/libauto.html>. Acesso em: 24 ago. 2000.
MILLARD, Maree. Tips and hints on library automation and automated library systems. [S. 1. : s. n., 1999]. Disponível em: <http://www.ilsr.com/ hints.htm>. Acesso em: 25 ago. 2000.

MILLER, Paul. Z39.50 for all. Ariadne, v. 21, Sept. 1999. Disponível em: $<$ http://www.ariadne.ac.uk/issue21/z3950/>. Acesso em: 26 ago. 2000.

REPORT on the 1998 evaluation of library software. Victoria : Department of Education, 1998. Disponível em: <http:// www.sofweb.vic.edu.au/itb/libryrpt/>. Acesso em: 26 ago. 2000.

ROWLEY, J. E. Guidelines on the evaluation and selection of library software packages. Aslib Proceedings, v. 42, n. 9, p. 225-235, Sept.1990.

SHAW, W.; CULKIN, P. B. Systems that inform: emerging trends in library automation and network development. Annual Review of Science and Technology, v. 22, p. 265-284, 1987.

TEBBETTS, Diane R. Automation in New England. [S. 1. : s. n., 1999]. Disponível em: <http://web.simmons.edu/ chen/nit/NIT'90/323tebbett.html>. Acesso em: 25 ago. 2000. 
Integração de todas as funções da biblioteca

Software em língua portuguesa

Possibilidade de customização (personalização) do sistema

Possibilidade de expansão ou inclusão de novos módulos sob demanda

Documentação (manuais)

Manuais com fluxos operacionais

Preço do produto

Interface gráfica

Possibilidade de customização (personalização) da interface

Menu de ajuda interativo

Arquitetura de rede cliente/servidor

Acesso via browser (Internet)

Acesso via Intranet

Velocidade de operação local (Intranet)

Velocidade de operação em rede (Internet)

Leitura de código de barras

Compatibilidade com o sistema operacional da biblioteca

Armazenamento e recuperação de caracteres da língua portuguesa

Data no formato $\mathrm{dd} / \mathrm{mmm} /$ aaaa (língua portuguesa)

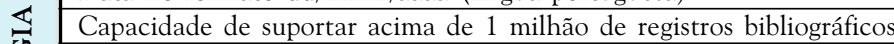

రु Atualização dos dados em tempo real

$\rightarrow$ Segurança na integridade dos registros

Z Possibilidade de identificar alterações feitas no sistema e os responsáveis

Compatibilidade com o formato MARC

Protocolo de comunicação Z39.50

Padrão ISO 2709

Disponibilização on-line do acervo (OPAC)

Importação e exportação de dados para alimentação de sistemas de catalogação cooperativa

Acesso on-line a catalogos coletivos

Acesso simultâneo de usuários

Acesso ilimitado de usuários

Número de licenças

Níveis diferenciados de acesso ao sistema (senhas)

Armazenamento e recuperação de documentos digitais em diversos formatos

Tratamento de texto e imagem conforme o DDIF (Digital Documentation Interchange Format)

Controle integrado do processo de seleção e aquisição

Integração dos dados de pré-catalogação da aquisição para o processamento técnico

Controle de listas de:

\begin{tabular}{l|l} 
& sugestão \\
\hline & seleção \\
\hline & aquisição \\
\hline & reclamações \\
\hline & recebimento
\end{tabular}

Controle de fornecedores

Controle de editores

Cadastro de entidades com as quais mantém intercâmbio de publicações

Mala direta de usuários, editoras e instituições com as quais a biblioteca mantém intercâmbio

Controle de assinatura de periódicos

Compatibilidade com o formato do CCN

Identificação de dados do processo de aquisição

Identificação da modalidade de aquisição (doação, compra, permuta, depósito legal)

Controle de datas de recebimento do material adquirido

Emissão de cartas de cobrança, reclamações e agradecimento de doações

Elaboração de lista de duplicatas

Identificação do usuário que sugeriu o título para aquisição

Controle da situação (status) do documento (encomendado, aguardando autorização, aguardando nota fiscal etc.)

Controle do orçamento

Possibilidade de especificação da moeda de transação 
Proposta de um método para escolha de software de automação de bibliotecas

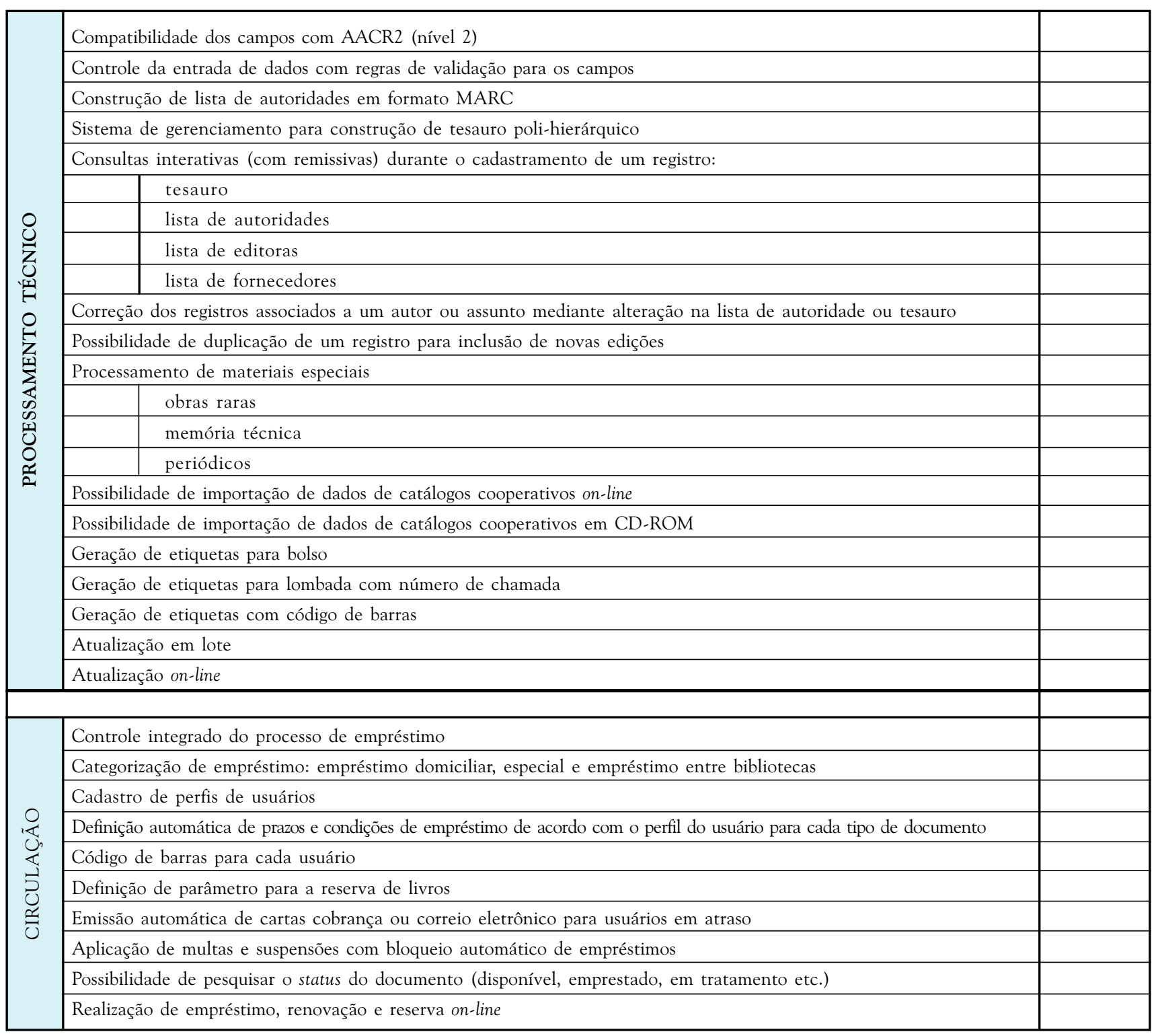


Lígia Café / Christophe Dos Santos / Flávia Macedo

Interface única de pesquisa (busca em todo o sistema)

Interface gráfica de pesquisa

Interface de busca avançada

Interface de busca on-line

Pesquisa nos campos:

autor
título
assunto
editor
local
palavras-chave
tipo de documento
resumo
classificação (CDU)
classificação (Tabela de Áreas do Conhecimento do CNPq)
ISSN
ISBN
idioma
número de chamada
data
todos os campos

Possibilidade de busca a partir de determinada data ou entre datas

Possibilidade de selecionar os campos a serem pesquisados por caixas de seleção

Possibilidade de selecionar o mesmo campo mais de uma vez

Refinamento da busca por:

frase

operador booleano AND

operador booleano NOT

operador booleano OR

truncamento à esquerda

truncamento à direita

truncamento ao meio

proximidade entre os termos

distância entre os termos

Possibilidade de busca a partir dos resultados

Possibilidade de salvar estratégias de buscas para utilização posterior

Busca automática por tesauro

Busca interativa a partir da seleção de termos do tesauro

Capacidade de ordenar e classificar os documentos pesquisados por:

autor

título

assunto

relevância

tipo de documento

data (ordem cronológica decrescente)

Apresentação das referências em ordem cronológica decrescente (default)

Possibilidade de limpar o formulario para nova pesquisa

Visualização do resultado da pesquisa em forma de referência bibliográfica breve ou completa (com resumo), de acordo com a ABNT

Visualização do resultado da pesquisa em forma de catálogo de acordo com a AACR2 (nível 2)

Visualização de todos os registros recuperados

Possibilidade de selecionar a quantidade de registros a serem exibidos em cada página

Visualização do número de registros recuperados

Visualização dos registros numerados (ex: 1/2, 2/2)

Capacidade de selecionar registros do resultado da pesquisa e imprimir

Capacidade de salvar os registros selecionados do resultado da pesquisa

Visualização do cabeçalho com identificação do assunto pesquisado e do número de referências dos registros gravados

Indicação do status do documento pesquisado (emprestado, em tratamento ou disponível)

Indicação do status do periódico pesquisado (corrente, encerrado, suspenso)

Possibilidade de solicitação de empréstimo do documento

Possibilidade de solicitação de cópias do documento pelo COMUT 
Proposta de um método para escolha de software de automação de bibliotecas

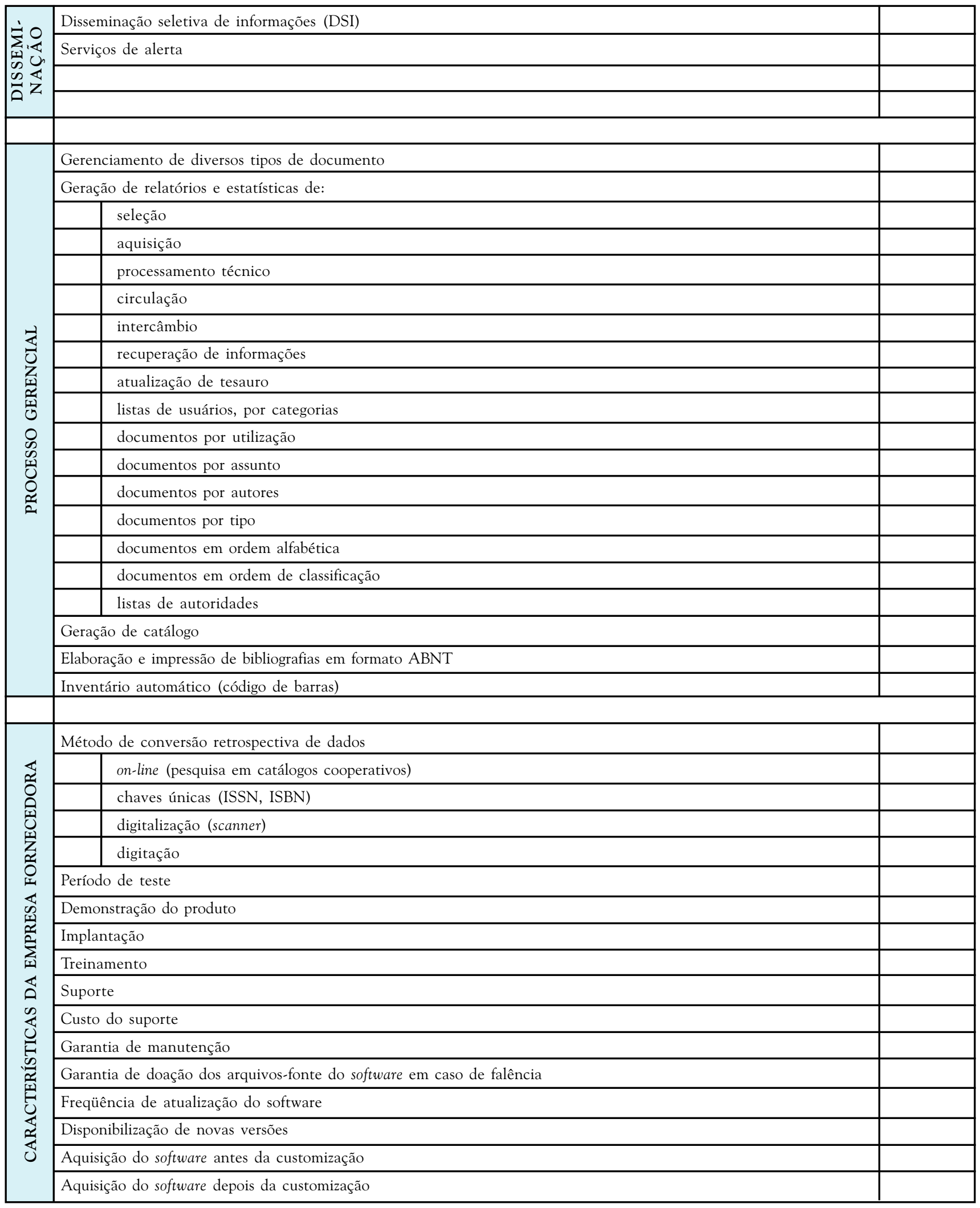

